Erica Monina

Gabriele Falzetti

Vincenzo Firetto

Laura Mariani

Claudio A. Caputi

\section{Behavioural evaluation in patients affected by chronic pain: a preliminary study}

Received: 14 April 2005

Accepted in revised form: 5 July 2006

Published online: 10 December 2006

E. Monina • L. Mariani

Psychological Management Structure,

Azienda Ospedaliero-Universitaria,

"Ospedali Riuniti Umberto I

G.M. Lancisi - G. Salesi”, Ancona, Italy

V. Firetto • C.A. Caputi

Operative Unit of Pain and Palliative Care,

Azienda Ospedaliero-Universitaria,

"Ospedali Riuniti Umberto I

G.M. Lancisi - G. Salesi”, Ancona, Italy

G. Falzetti

Department of Neurosciences,

Section of Anaesthesia

and Intensive Care Unit,

Azienda Ospedaliero-Universitaria,

"Ospedali Riuniti Umberto I

G.M. Lancisi - G. Salesi”, Ancona, Italy

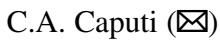

Unità Operativa Medicina

del Dolore e Palliativa,

Azienda Ospedaliero-Universitaria,

"Ospedali Riuniti Umberto I

G.M. Lancisi - G. Salesi”, Via Conca 71,

I-60020 Torrette di Ancona, Italy

e-mail: c.caputi@ao-umbertoprimo.marche.it

Tel.: +39-71-5963152

Fax: +39-71-5963140

\begin{abstract}
The aim was to analyse the existing relation between a subjective evaluation of pain with the use of the Verbal Numerical Scale (VNS) and an objective behavioural measure associated with pain, by means of the Pain Behaviour Rating Scale (UAB). An observational correlation study was carried out in a hospital environment. The study included 61 patients affected with multiple forms of non-malignant chronic pain; the behaviour was observed by the nursing staff. In general, a positive but moderate correlation was obtained between VNS and UAB scales $(r=0.29, p<0.0001)$. Observing behaviour and listening to the patient constituted two complementary and non-interchangeable methods for assessing the level of pain capable of providing a global and objective portrayal of the pain experience.
\end{abstract}

Keywords Pain - Pain behaviour observation - Pain intensity selfreport

\section{Introduction}

The International Association for the Study of Pain (IASP) defines pain as "an unpleasant sensory and emo- tional experience associated with actual or potential tissue damage or described in terms of such damage" [1]. This definition emphasises how the perception of pain may have not only a biological/structural foundation but may also derive from psychological factors, such as the per- 
sonality of the subjects, their cognitive-emotional characteristics, family role models, etc., which inevitably influence the perception and description of the experience.

Description of pain by the patient can be attributed to an underlying physical basis, but can often also be psychological or legal in nature. The assessment of a strictly personal experience like pain should, therefore, foresee the close examination of other aspects: history of the patients and their experience with pain, direct observation of their behaviour, and analysis of specific physiological parameters.

Verbal description of pain intensity and real painlinked behaviour are two different evaluation systems, which patients should use to communicate their suffering.

Behavioural investigation is an aspect that should not be neglected in the evaluation of patients affected by chronic pain, in that it not only conditions the clinical diagnosis orienting it towards appropriate therapeutic choices but also verifies the reliability of what has been described by the patients $[2,3]$.

The literature reports little correspondence between what has been reported regarding the intensity of the pain perceived by patients and what can be deduced by their caregivers [4, 5].

It is not difficult to imagine the usefulness of monitoring behaviour in the case of children or the elderly, who are potentially more exposed to communication problems, and could find it difficult or impossible, because of cultural or personal reasons, to express an opinion in regard to one's experience of pain or filling out descriptive questionnaires.

The behaviour expressed during pain may include complaints, drug use, or the search for a treatment, physical or social disability, and behaviour tied to physical pain, such as facial grimaces, moans or massage of the painful area. Many of these actions can be objectively analysed, quantified [6-10], and compared with the description that the patients themselves reported regarding their pain experience [11, 12].

Several studies have found a low or moderate correlation between an objective measure of the patients' global behaviour and a subjective measure of the perceived pain intensity [13-17]. It was also observed that the strength of the correlation in question could depend on various factors: the chronicity of the pain, the moment in which the subjective evaluation was carried out, and the type of pain behaviour observed and tested [9].

\section{Materials and methods}

\section{Objective}

The aim of this study was to observe and investigate the relation between a subjective pain evaluation by the patient and an objective measure of the patient's actual behaviour. Is the patients' judgement of their pain in accordance with what they manifest from a behavioural point of view?

\section{Participants}

After obtaining informed consent, patients (range 25-85 years) affected by chronic non-malignant pain ranked in classes, without other associated pathologies that can increase pain (diabetes, major depression in treatment, pathologies with known diagnosis and currently being treated), and with good health status (ASA Physical Status 1-2), capable of understanding the questions for VNS assessment, hospitalised from March to July 2004 at the Pain Therapy and Palliative Care Unit were enrolled in the study. Therapy was based on the type of diagnosed pain and was the same for each pain category identified.

\section{Monitoring and data collection}

The subjective perception of pain was investigated with the use of the Verbal Numerical Scale (VNS), an instrument of easy administration, in which the patient is asked to choose a number from 0 to 10 to represent the intensity of pain felt.

For VNS evaluation, patients were asked to score their pain from 0 to 10 , where 0 was no pain and 10 was the worst pain experienced, or "what is the degree of pain you experienced from 0 to 10 at this moment, if 0 means no pain and 10 is the worst pain you have experienced?" [18].

The choice of using this scale instead of the common Visual Analogue Scale (VAS) arose from the necessity to monitor a sample of prevalently older patients, who would have found it difficult to complete the VAS questionnaire. The VNS eliminated, in fact, the necessity of visual and motor coordination requested when administering the VAS, therefore offering a greater possibility of being completed [18-20]. It has also been demonstrated that VNS scores are comparable with Numeric Rating Scale (NRS) scores [21].

Behavioural assessment was carried out with the use of the UAB Pain Behaviour Scale [4], a standardised instrument capable of assessing through direct observation the global behaviour of the patient [8]. The scale consists of 10 target behaviours, each of which contributes equally to the total score, providing a range of possible scores from 0 to 10 . This set of target pain behaviours was selected from a larger list and represented to the UAB Authors some of the most salient, reliably measurable and frequently observed pain behaviours in a chronic pain population: verbal and non-verbal vocal complaints, time spent lying down because of pain, facial grimaces, quality of standing posture and mobility, suffering body language, use of visible supportive equipment, stationary movements (how often the patient shifts position while sitting or standing) and pain medication behaviour. Ratings were based on frequency estimates for both of these variables: absent (0), occasional (1/2) and frequent (1).

Patients were monitored for 5 consecutive days: on admission (day one), VNS and UAB scores were collected for a pretherapeutic baseline evaluation. Over the subsequent four days, 
VNS and UAB scores were always collected from the patients upon awakening $(8: 00 \mathrm{~h})$ by the same person to evaluate the benefit of therapy from the previous day and the progressive improvement in the clinical picture.

During the day, behavioural observation and consequent completion of the UAB scale was carried out by the nursing staff, who seemed better suited to observe certain behaviours of hospitalised patients, as they were more often in contact with them. The UAB was administered three times daily for 5 consecutive days. The decision to evaluate the patients 3 times a day coincided with the necessity to observe their behaviour during the entire day rather than in one precise moment; the three evaluation times involved different observers. The nursing staff was quickly trained at the start of the study to compile UAB scores on the hospitalised patients who were enrolled in the study, and although their knowledge of the UAB was poor, it was the same for each member of the paramedical personnel, who only had to compile the UAB tables. The nursing staff's work schedule was based on shift rotations; thus, the nurse who administered the UAB was always different during the day and during the study period, and the same nurse was never responsible for the entire weekly afternoon or evening $\mathrm{UAB}$ scoring during the study period.

The therapy for these patients was administered only in the morning according to internal therapeutic protocols, which were the same based on the type of pain; the nurse observers could not dispense pain medication to the patients without medical prescription. Internal therapeutic protocols were rigorously respected during the study, and pain medication remained constant during the five-day period.

Patients hospitalised for more or less than 5 days were excluded from the study.

\section{Statistical analysis}

Data are shown in mean and standard deviation (SD) intervals.

The differences between VNS and UAB in the pain groups and the UAB differences for each day were analysed with repeated two-way ANOVA. Correlation coefficients were calculated with $95 \%$ confidence intervals (CI) for the whole study population, for the subgroup with lumbosciatalgia (in its entirety and ranked by gender), and for days one and five of the study. Correlation coefficients for days one and five for males and females in the subgroup with lumbosciatalgia were compared with an unpaired $t$-test, while clinical progress from day one to five, based on VNS and UAB scores, was compared with paired $t$-test. Linear regression curves were compared for days one and five.

Statistical analysis was performed with SPSS 13.1 for Windows program (SPSS Inc., Chicago, IL). p-values $<0.05$ were considered statistically significant.

\section{Results}

A total of 61 patients, with good health status (Mean ASA - PS 1.31, SD 0.23) were enrolled in the study: 20 males $(33 \%)$ and 41 females $(67 \%)$.
Six groups were sorted based on pain type, without other associated pathologies that can increase pain, with the following distribution: (a) lumbosciatalgia, 26 patients (44\%); (b) headache; (c) cervicobrachialgia; (d) neuralgia, 7 patients $(11 \%)$ in each group; (e) cruralgia, 5 patients (8\%); (f) other types of pain, 9 patients $(15 \%)$.

In Figure 1, the distribution of subgroups based on pain presentation, gender and age is shown in absolute values. It is evident that in the lumbosciatalgia group, male and female distribution is similar, whereas in the other groups, there is a female prevalence. Mean age for the 61 patients was 63 years (SD 11); it is evident that in the headache group, the age is lower than the mean.

Figure 2 and Table 1 report VNS and UAB presentation values on admission (first day values) in the six study groups. VNS values are similar, while there are statistical differences $(p<0.01$, ANOVA) in UAB values. The greatest differences were also found for headache between VNS and UAB values. The limitation of these data is that they were generated from a small sample size $(n=7)$; these results should be analysed with other accurate studies.

In Figure 3 VNS and UAB trends are shown for the entire study population from day one to five with significant reduction in VNS trends $(p<0.05$ paired $t$-test for continuous data) not reflected by the UAB trend.

In Figure 4 linear regression curves for VNS and UAB on days one and five are shown. This complex figure shows the correlation between VNS and UAB evaluated on the morning of day one in grey and the correlation between VNS and UAB evaluated on the morning of day five with black triangles. It is evident that the grey points are more scattered than the black triangles, which is confirmed by 95\% CI from the fit line. In fact, the $95 \%$ CI for the fit line on day one (in grey, $r^{2}=0.012 ; \mathrm{CI},-0.092-0.431$, non-significant) is larger than that on day five (black fit line, $r^{2}=0.423$; CI: $\left.0.331-0.638, p<0.0001\right)$.

Figure 5 shows mean and SD UAB values recorded at three specific daily times on the five study days. There are statistically significant differences between morning, afternoon and evening $(p<0.005$, day one; $p<0.001$, day two; $p<0.01$, day three; $p<0.05$, days four and five; repeated measures two-way ANOVA), which nevertheless gradually decrease. It is evident that UAB evening values are significantly lower than the other daily times.

In Table 2, the Pearson correlation and 95\% CI for coefficient B are shown for the entire study population. Correlation between VNS and UAB for the entire study population was $0.284(95 \%$ CI for B, 0.123-0.366, N. 61*5) with $p<0.0001$. Also in Table 2, the Pearson correlation and 95\% CI for coefficient B are shown for days one and five of the study, and it is evident that the correlation progressively increases with time: from $0.112(95 \% \mathrm{CI}$ for $\mathrm{B}$, $-0.092-0.231 ; p=0.392$, N. $61 * 5)$ on day one to $0.634(95 \%$ CI for B, $0.331-0.738 ; p<0.0001$, N. $61 * 5)$ on day five. 


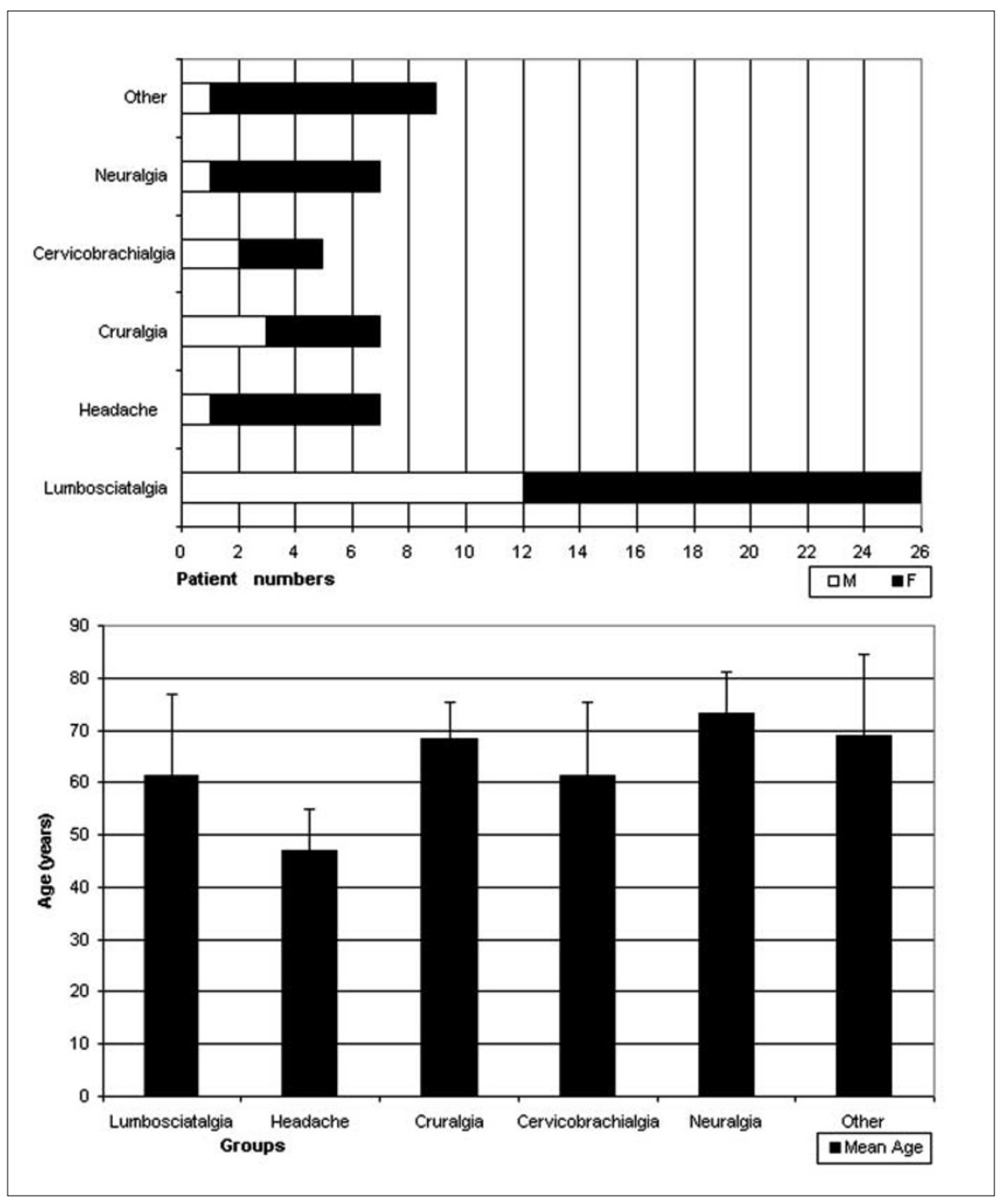

Fig. 1 Gender distribution in absolute values ( $\mathrm{M}$, male; $\mathrm{F}$, female) (upper) and age distribution in mean and standard deviation in subgroups (lower)

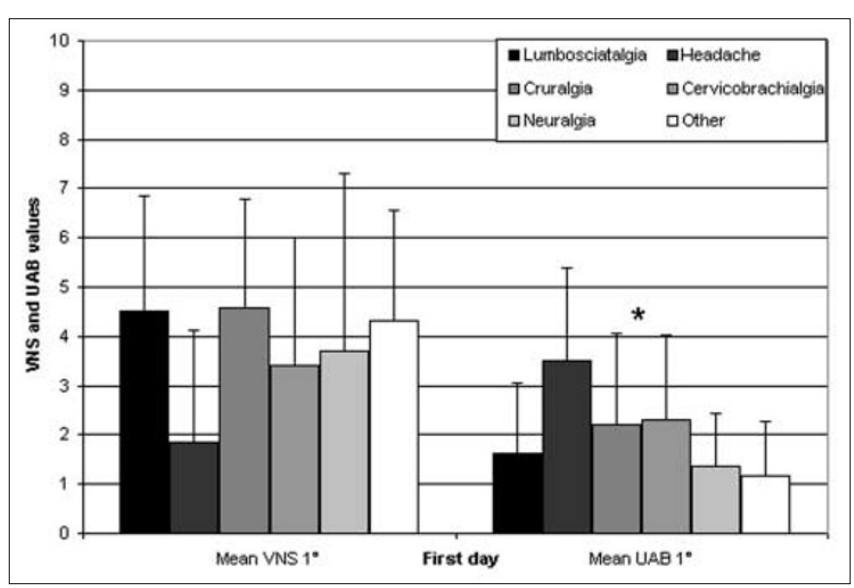

Fig. 2 Verbal Numerical Scale (VNS) and Pain Behaviour Rating Scale (UAB) distribution on day one (presentation values) in mean and standard deviation in considered pain groups $* p<0.01$, ANOVA
Based on the size of the lumbosciatalgia subgroup, the same statistical analysis was restricted to this group, which showed the same number of males and females (Fig. 1). Table 2 shows the Pearson correlation and 95\% $\mathrm{CI}$ for coefficient B in the lumbosciatalgia group by gender distribution. Correlation in the lumbosciatalgia group was 0.268 (95\% CI for B, 0.066-0.290, N. 26*5), with $p=0.002$. The correlation in women in this group was smaller than that in men, respectively, 0.241 (95\% CI for B, $0.005-0.368$, N. $14 * 5, p=0.044) v s .0 .321$ (95\% CI for B, $0.039-0.404, p=0.012$, N. $12 * 5$ ). Correlation coefficients compared with paired $t$-test did not present significant differences.

Figure 3 shows VNS and UAB trends for the lumbosciatalgia group compared with the same trends for the entire study population. The VNS index presented a more significant reduction $(p<0.01$ paired $t$-test for repeated measures) than the index of the study population $(p<0.05)$. 
Table 1 Verbal Numerical Scale (VNS) and Pain Behaviour Rating Scale (UAB) values in mean and standard deviation on the five days of study

\begin{tabular}{lrrrr}
\hline Days & Mean VNS & SD VNS & Mean UAB & SD UAB \\
\hline 1st day & 4.00 & 2.54 & 1.86 & 1.59 \\
2nd day & 3.19 & 2.29 & 1.89 & 1.38 \\
3rd day & 3.10 & 2.03 & 2.04 & 1.53 \\
4th day & 2.49 & 1.98 & 1.67 & 1.46 \\
5th day & 2.15 & 1.89 & 1.61 & 1.44 \\
\hline
\end{tabular}

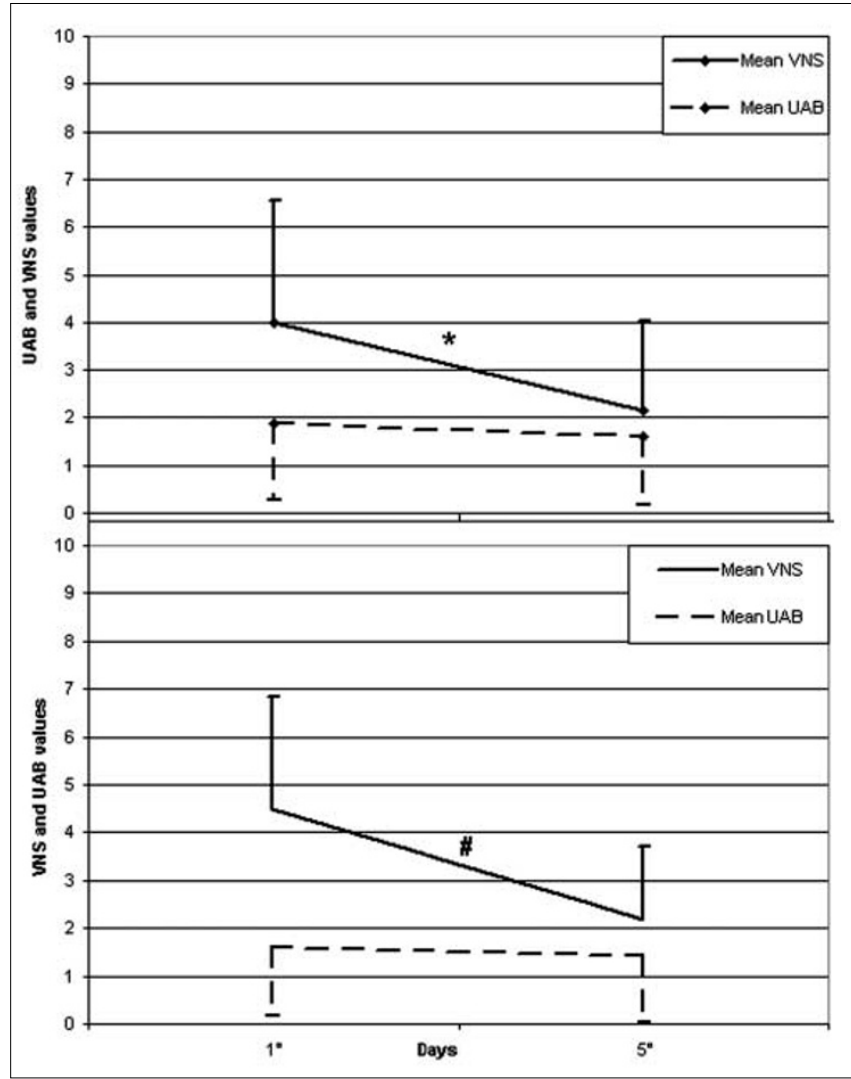

Fig. 3 Verbal Numerical Scale (VNS) and Pain Behaviour Rating Scale (UAB) trend from day one (pain presentation) to day five (end of study) in the total group (upper $* p<0.05$, repeated measurement paired $t$-test) and in the lumbosciatalgia group (lower ${ }^{\#} p<0.01$, repeated measurement paired $t$-test)

Also, in this analysis, UAB did not show a statistically significant reduction.

\section{Discussion}

Measurement of the pain experience is useful for several reasons: it provides the starting point from which future

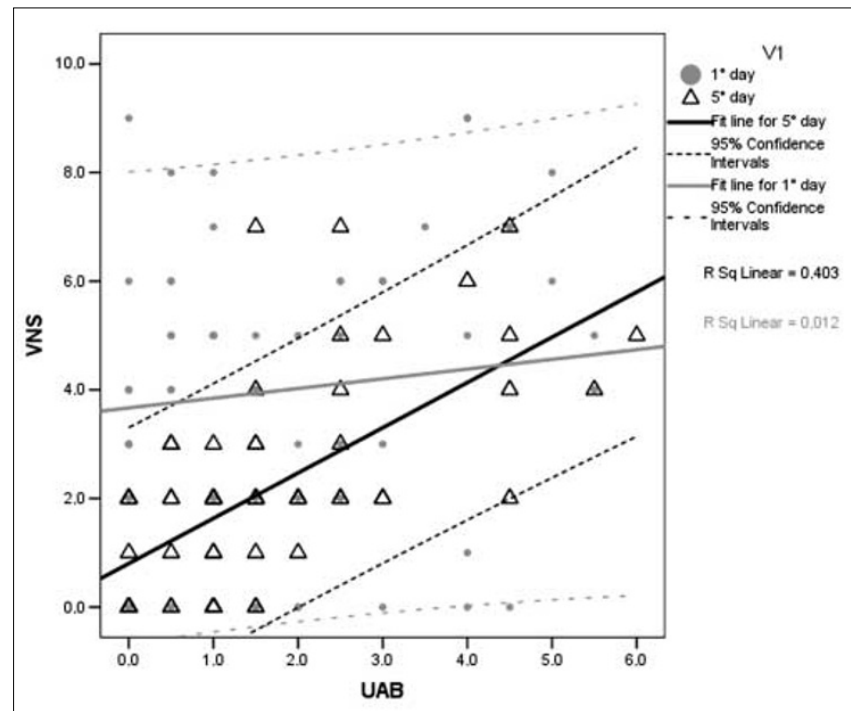

Fig. 4 Quadratic regression curve prepared with Verbal Numerical Scale (VNS) and Pain Behaviour Rating Scale (UAB) values of the total group on day one (pain presentation day) and on day five (end of study) $\left(r^{2}=0.012 ; 95 \%\right.$ CI for B, $-0.192-0.431 ; p=0.392$ for day one, $r^{2}=0.403 ; 95 \% \mathrm{CI}$ for $\mathrm{B}, 0.331-0.638 ; p<0.0001$ for day five)

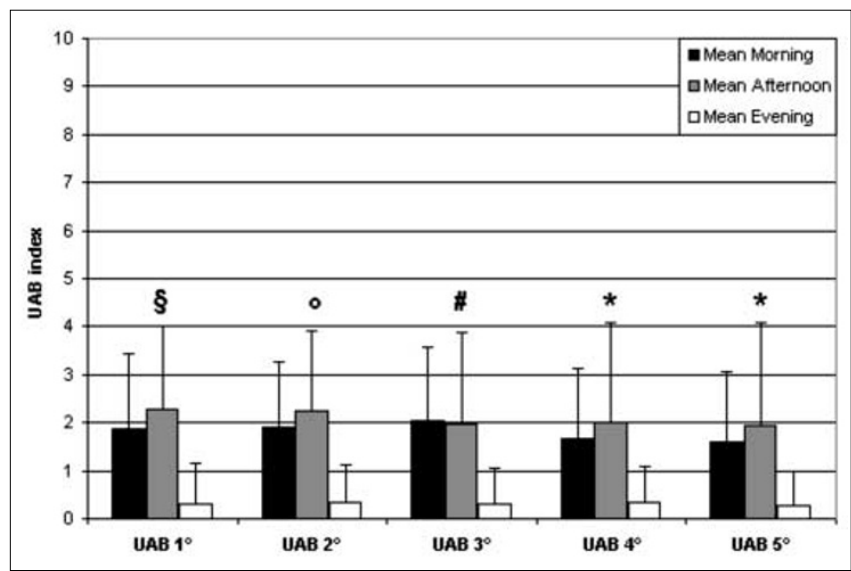

Fig. 5 Pain Behaviour Rating Scale (UAB) values in mean and standard deviation on three daily evaluations during the five days of study. $\S p<0.005 ;{ }^{\circ} p<0.001$; $\# p<0.01 ;{ }^{*} p<0.05$; ANOVA

therapeutic interventions can be considered; it reveals the degree of impairment and disability of the patient; it differentiates a subject who is really suffering from one who feigns; and it assesses the influence personality may have on pain expression.

The subjective/objective approach to chronic pain seems to be extremely valid, in that, in addition to examining the subjective perception of pain with self-reporting instruments, it evaluates the degree of invalidity and the behaviour associated with pain by way of behavioural observation. Are what patients refer compatible with what they do? 
Table 2 Pearson correlation (beta factor), 95\% CI for coefficient B and significance ( $p$ ) between Verbal Numerical Scale (VNS) values considered as independent variable and Pain Behaviour Rating Scale (UAB) values as dependent variable in the total group; on days one and five, in the lumbosciatalgia group (total and categorised for gender)

\begin{tabular}{lccc}
\hline Correlation indices & $\begin{array}{c}\text { Pearson } \\
\text { correlation beta }\end{array}$ & 95\% CI for coefficient B & \\
\hline $\begin{array}{l}\text { Independent variable: VNS in group total } \\
\text { Group UAB }\end{array}$ & 0.294 & 0.123 & 0.266 \\
$\begin{array}{l}\text { Independent variable: VNS 1st day } \\
\text { UAB 1st day }\end{array}$ & 0.112 & -0.092 & 0.231 \\
VNS 5th day & 0.635 & 0.331 & 0.638 \\
UAB 5th day & & 0.290 \\
$\begin{array}{l}\text { Independent Variable: VNS in lumbosciatalgia group } \\
\text { Group UAB }\end{array}$ & 0.268 & 0.066 & 0.0001 \\
VNS for males in lumbosciatalgia group & & & 0.304 \\
UAB males & 0.322 & 0.039 & 0.002 \\
VNS for females in lumbosciatalgia group & & & 0.392 \\
UAB females & 0.241 & 0.005 & 0.012 \\
\hline
\end{tabular}

The association between VNS and UAB scores found by our analysis was positive but rather low $(0.294$, $p<0.0001)$; this fact seems to confirm what was found by previous studies $[11,12]$, and it shows that self-reporting pain intensity and pain behaviour constitute different aspects of the complex pain experience. The small sample size and heterogeneous class of pain analysed are limitations to this study.

During the five days of assessment (from days one to five of evaluation), we observed a progressive increase in correlation between VNS and UAB (Table 2). This result corresponds with what is shown in Figure 3 when, on day five, the mean distance between VNS and UAB scores decreased substantially, almost to the point of overlapping.

Therefore, although patients described a lower pain intensity, probably because of therapy, their pain behaviour was not very different from their previous pain behaviour, when they showed a greater pain intensity. It should be remembered that administered therapy is only palliative for pain treatment and does not treat the specific underlying causes.

This fact could indicate that UAB is an unsuitable instrument for pain behaviour evaluation in the long-term, or, from a psychological point of view, we can hypothesise that the expectations shown by patients on admission to hospital play a substantial role in their amplification of pain perception. It would be interesting to observe the trend of the two measures over a longer time period to verify their stability.

Analysing VNS and UAB scores in six subgroups (six different groups for six different pathologies without specific treatment) during the first day of assessment, we observed that while VNS scores were very similar (almost overlapping), a significant difference existed between UAB scores (Fig. 2); these scores seemed to underestimate the pain of patients expressed with VNS in all groups. The different type of pain focused on in this study is a limitation because heterogeneity plays an important role in the perception of pain by patients. In particular, we found a trend inversion for the headache group, in which UAB scores were higher than VNS scores, overestimating in this case the pain perception intensity expressed with VNS for this group. This aspect cannot be further analysed in this study due to the small sample of patients with headache, but warrants further study.

As mentioned above, the UAB scores related to patient pain behaviour during hospitalisation were collected at three different moments of the day by different nursing staff both during the day and during the five days of the study. We found significant differences between the scores obtained at different observation times (morning, afternoon, evening), which tended to decrease during the hospitalisation period, showing that day therapy is effective not only in the short term but also in the long term, that is, from days one to five. The UAB evening scores were, from a statistical point of view, lower in respect to other scores. Considering, therefore, that UAB trends did not show changes during the five days of assessment, we could hypothesise that this scale was more useful in evaluating patients at different times of the day, thus avoiding having to ask them each time direct questions on how they felt, whereas the VNS could more likely be useful in evaluating therapeutic effects over time, day by day.

The second part of our study was dedicated to analysing the correlation between UAB and VNS scores 
in the lumbosciatalgia group, as this group was more numerous and homogenous (in relation to gender and age distribution) than the others.

In the lumbosciatalgia group, the trend in VNS and UAB scores was very similar to that of the total study population (Fig. 3), but with a statistically significant greater reduction in VNS scores compared with those of the whole sample.

The correlation between VNS and UAB scores in the female group was weaker and also less significant compared to the male group, even though the lack of significance between correlation coefficients by $t$-test identified the same low correlation between scores in males and females in the lumbosciatalgia group, which, moreover, was very similar to results from the total sample. These results were in contrast to those found in previous studies regarding the differences in pain perception and in discrimination of pain sensations between genders. According to past studies, women reported more severe and frequent pain, showed lower pain thresholds and pain tolerance than men, and seemed more capable of discriminating pain sensations [21-28]. Recent reviews [29, 30] suggest that different pain expressions found between men and women do not necessarily reflect physiological/anatomical differences between the two sexes, but could be related to cognitive or psychosocial factors (for example, the more or less marked and culturally acquired adherence to stereotypes typical to female and male social roles, and expectations tied to the gender role, etc.), which inevitably influence perception and manifestations of suffering.

The absence of significant differences between males and females in the pain experience observed in our research could be related to the fact that, in conditions of clinically severe pain, sexual differences in perception and expression of pain tend to reduce substantially.

In conclusion, the involvement of nursing personnel in monitoring behaviour has been found to be an extremely valid method, not only in collecting particular expressions of the patients at different times of day but also in integrating clinical practice with continued observation of the patients' conditions.

Realisation of a more reliable and accurate observation of behaviour would have required, however, appropriate training associated with intensive practice; the major limitation of this study concerns the absence of prior thorough training for the observers and not simply a compilation of one set of tables. This aspect will therefore be considered more adequately in future studies.

\section{Conclusions}

The results of this study, in light of its limitations, underscore the fact that the behaviour of patients partially reflects their description of pain. Behavioural observation and listening to the patients constitute complimentary and non-interchangeable methods of assessing pain, capable of providing a general representation of the objective conditions of the patients, their pain behaviour and the necessary treatments. Self-reported pain intensity is a reliable indicator for the assessment of clinical pain and cannot be replaced by pain behaviour observation; vocal complaints, facial grimaces and body language suggest the presence of pain but do not reflect the extent of pain, which can be evaluated only considering in primis the patients' self-report.

VNS and UAB scales constitute two complementary instruments for analysing subjective and objective dimensions of pain. Statistical analysis shows a gradual decrease in VNS scores each day of the study period, unlike the trend in UAB scores, which showed no significant differences between days one and five. However, considering the significant differences found among the three daily UAB scores on each day of the study and the fact that patients should not be further disturbed to complete these evaluations, it seems appropriate to propose $\mathrm{UAB}$ as a daily evaluation instrument. In contrast, given the significant difference between days one and five for VNS scores, we propose VNS for pain evaluation on a day-to-day basis.

In the lumbosciatalgia subgroup, we did not observe significant differences in pain expression between males and females. This result seems to contradict the hypothesis of other researchers concerning possible gender differences in pain perception and expression.

\section{References}

1. Watt-Watson JH, Ivers Donovan M (1992) Pain management, nursing perspectives. Mosby Year Book, St. Louis, MO

2. Kent G (1985) Memory of dental pain. Pain 21:187-194
3. Melzack R, Torgerson WS (1971) On the language of pain. Anesthesiology 34:50-59
4. Nekolaichuk CL, Bruera E, Spachynski K et al (1999) A comparison of patient and proxy symptom assessments in advanced cancer patients. Palliat Med 13:311-323 
5. Hall-Lord ML, Larsson G, Steen B (1998) Pain and distress among elderly intensive care unit patients: comparison of patients' experiences and nurses' assessments. Heart Lung 27:123-132

6. Follick M, Ahern D, Aberger E (1985) Development of an audiovisual taxonomy of pain behavior: reliability and discriminant validity. Health Psychol 4:555-568

7. Keefe FJ, Block A (1982) Development of an observation method for assessing pain behavior in chronic low back pain patients. Behav Ther 13:363-375

8. Richards JS, Nepomuceno C, Riles M, Suer Z (1982) Assessing pain behavior: the UAB Pain Behavior Scale. Pain 14:393-398

9. Vlaeyen JW, Pernot DF, Kole-Snijders AM et al (1990) Assessment of the components of observed chronic pain behavior: the Checklist for Interpersonal Pain Behavior (CHIP). Pain 43:337-347

10. Waddell G, Richardson J (1992) Observation of overt pain behaviour by physicians during routine clinical examination of patients with low back pain. J Psychosom Res 36:77-87

11. Labus JS, Keefe FJ, Jensen MP (2003) Self-reports of pain intensity and direct observation of pain behavior: when are they correlated? Pain 102:109-124

12. McCahon S, Strong J, Sharry R, Cramond T (2005) Self-report and pain behavior among patients with chronic pain. Clin J Pain 21:223-231
13. Baumstark KE, Buckelew SP, Sher KJ et al (1993) Pain behavior predictors among fibromyalgia patients. Pain 55:339-346

14. Buckelew SP, Parker JC, Keefe FJ et al (1994) Self-efficacy and pain behavior among subjects with fibromyalgia. Pain 59:377-384

15. Koho P, Aho S, Watson P, Hurri H (2001) Assessment of chronic pain behaviour: reliability of the method and its relationship with perceived disability, physical impairment and function. J Rehabil Med 33:128-132

16. Ohlund C, Lindstrom I, Areskourg B et al (1994) Pain behavior in industrial subacute low back pain. Part I. Reliability: concurrent and predictive validity of pain behavior assessments. Pain 58:201-209

17. Weiner D, Pieper C, McConnell E et al (1996) Pain measurement in elders with chronic low back pain: traditional and alternative approaches. Pain 67:461-467

18. Jensen MP, Karoly P, Braver S (1986) The measurement of clinical pain intensity: a comparison of six methods. Pain 27:117-126

19. Ferraz MB, Quaresma MR, Aquino LR et al (1990) Reliability of pain scales in the assessment of literate and illiterate patients with rheumatoid arthritis. J Rheumatol 17:1022-1024

20. McCormack HM, Home DJ, Sheather S (1988) Clinical applications of visual analogue scales: a critical review. Psychol Med 18:1007-1019
21. Paice JA, Cohen FL (1997) Validity of a verbally administered numeric rating scale to measure cancer pain intensity. Cancer Nurs 20:88-93

22. Dao TT, LeResche L (2000) Gender differences in pain. J Orofac Pain 14:169-184

23. LeReche L (2000) Epidemiologic perspectives on sex differences in pain. In: Fillingim RB (ed) Sex gender and pain. IASP Press, Seattle, pp 223-249

24. Rollman GB, Lautenbacher S, Jones KS (2000) Sex and gender differences in responses to experimentally induced pain in humans. In: Fillingim RB (ed) Sex, gender and pain. IASP Press, Seattle, pp 165-190

25. Jackson T, Iezzi T, Chen $\mathrm{H}$ et al (2005) Gender, interpersonal transactions, and the perception of pain: an experimental analysis. J Pain 6:228-236

26. Unruh AM (1996) Gender variations in clinical pain experience [Review] [235 refs]. Pain 65:123-167

27. Soetanto AL, Chung JW, Wong TK (2004) Gender differences in pain perception: a signal detection theory approach. Acta Anaesthesiol Taiwan 42:15-22

28. Vallerand AH, Polomano RC (2000) The relationship of gender to pain. Pain Manag Nurs 1[Suppl 1]:8-15

29. Wise EA, Price DD, Myers CD et al (2002) Gender role expectations of pain: relationship to experimental pain perception. Pain 96:335-342

30. Myers CD, Riley JL 3rd, Robinson ME (2003) Psychosocial contributions to sex-correlated differences in pain. Clin J Pain 19:225-232 